\author{
Anđelka Ž. Krstanović* \\ Universität von Banja Luka \\ Fakultät für Philologie \\ Institut für Germanistik
}

\title{
LITERATURKRITIK ONLINE
}

\section{EINE ANALYSE DER WERTENDEN KRITERIEN AUF DEUTSCHEN UND SERBISCHEN INTERNETPORTALEN**}

\author{
Original wissenschaftliche Arbeit \\ UDC 82:004.773 \\ https://doi.org/10.18485/kkonline.2020.11.11.8
}

\begin{abstract}
Der Gegenstand der vorliegenden Arbeit sind Literaturkritiken auf deutschen und serbischen Internetportalen, die in dieser Onlineform in den letzten zwei Jahrzehnten überhandnahmen und dadurch den Literaturkritiken in Printmedien, die immer häufiger gekürzt und weniger gedruckt werden, einen Schritt voraus sind. Die Literaturkritiken auf den ausgewählten Portalen werden durch analytische und komparative Methode in Bezug auf wertende Kriterien hinterfragt, die für eine gute Rezension bürgen und bei der Besprechung von literarischen Texten angewandt werden. Es wird überprüft, ob und in welchem Maße auf den Portalen autorisierte Texte erscheinen, ob die unterzeichneten Namen die Fachkompetenz vorweisen und in den veröffentlichten Texten die wissenschaftlichen Kriterien bevorzugt werden, ob ferner die fachlichen Kompetenzen keinen externen Faktoren, wie dem Druck von Verlagshäusern und marktorientierten Geschäftsregeln, untergeordnet werden. Schließlich wird hinterfragt, in welchem Maße auch die Rezensionen einem weiteren Begutachtungsverfahren als Kontrollmechanismus unterzogen werden. Die Analyse der genannten Faktoren soll dazu beitragen, einen besseren Überblick hinsichtlich der Erhaltung der Voraussetzungen für eine gute Literaturkritik auf den Internetportalen zu verschaffen.
\end{abstract}

Schlüsselwörter: Literaturkritik online, Blogs, Foren, Online-Zeitschriften und Magazine, Buchhandlungsportale, literaturkritische Portale.

Der thematische Schwerpunkt in diesem Beitrag ist die Online-Literaturkritik. Die Ent- scheidung, sich gerade mit dieser Art der medialen Vermittlung der Literaturkritik zu beschäftigen, geht vor allem aus ihrer Aktualität und Progression hervor. Die literaturkritischen Texte als Online-Publikationen erleben seit den 1990er-Jahren, insbesondere im letzten Jahrzehnt, einen Aufschwung. Sie sind in unzähligen Varianten dem Nutzer bzw. Leser zugänglich, und man kann bei einer unüberschaubaren Zahl der verschiedenen Arten von Buchbesprechungen sehr schnell den Überblick verlieren.

\footnotetext{
* Filološki fakultet, ul. Petra Bojovića 1A, 78000 Banja Luka, Republika Srpska, Bosna i Hercegovina; email: andjelka.krstanovic-jankovic@flf.unibl.org.

** Der vorliegende Beitrag wurde in verkürzter Form bei der 1. Internationalen Tagung für Angewandte Germanistik im Juni 2017 in Bukurest präsentiert.
} 
Es wird fast unmöglich, auf den ersten Blick eine Selektion in Bezug auf die Qualität der dargebotenen Online-Plattformen zu treffen. So ist dieser Beitrag zur OnlineLiteraturkritik auch als ein Teil der globalen Diskussion zum Thema der Vorteile und Nachteile des Internets zu erfassen.

Die Literaturkritik hat eine jahrhundertelange Tradition, die festeren Umrisse als Disziplin bekommt sie im 18. Jahrhundert, in welchem sie u. a. durch Beiträge von Gottsched, Lessing und Herder kanonisiert wird, um nur einige Namen zu nennen. Ihr Medium im 18. und 19. Jahrhundert waren vor allem Zeitschriften und Zeitungen. Man sei an dieser Stelle an Gottscheds Neuer Büchersaal der schönen Wissenschaften und freyen Künste (1745-1750) erinnert, wie auch Lessings literaturkritisches Unternehmen Briefe, die Neueste Literatur betreffend (bis 1765) und Friedrich Nicolais Allgemeine Deutsche Bibliothek (1765-1806).

Im 20. Jahrhundert erfüllen diese Rolle die Tageszeitungen ( $F A Z$, Neue Zürcher Zeitung etc.) und Magazine (z. B. Der Spiege/). Die Druckmedien veröffentlichten Rezensionen, deren Verfasser einem Kontrollmechanismus in Bezug auf Auswahl der Bücher und Qualität der Texte seitens der Kulturredakteure unterzogen waren. Diese wiederum nahmen eine Erstselektion vor, nachdem sie die freien Exemplare von Verlagshäusern zugeliefert bekommen hatten. Später fanden auch andere Medien Eingang in die Rezensionswelt, so Hörfunk und Fernsehen. Seit den 1990er-Jahren erlebt die Online-Kritik einen Boom, wogegen die Printmedien, in denen die Rezensionen immer weniger gedruckt und immer mehr gekürzt werden, den Kampf um die Konkurrenz verlieren.

Die Literaturkritik als Teil des literarischen Systems war schon immer unentbehrlich für das gesamte literarische Leben. Nach den Literaturwissenschaftlern Thomas Anz und Rainer Baasner ist die Literaturkritik

[...] eine der wichtigsten Vermittlungsinstanzen zwischen Literatur und Lesern [...] Literaturkritik ist omnipräsent: Selbst wer grundsätzlich keine Rezensionen liest, ist über Urteile anderer den Wirkungen der Kritik ausgesetzt. Sie ist eine Institution der literarischen Öffentlichkeit, die individuelle und kollektive Vorstellungen darüber prägt, was Literatur ist, sein kann oder sein sollte und wie einzelne Texte einzuschätzen sind. Sie verschafft Überblicke, wählt aus, informiert, interpretiert, wertet und regt dazu an, über Literatur zu diskutieren (Anz und Baasner, 2007).

Die Literaturkritik hatte schon immer einen klaren Adressaten - den Leser. Ihre klaren Aufgaben sind: Selektion, Information, Interpretation und Wertung von literarischen Werken. Und für ihre Qualität bürgte seit je eine klare Bedingung - die Gelehrsamkeit, bzw. eine hohe Fachkompetenz der Rezensenten, die vor allem aus 
der Literaturwissenschaft und Ästhetik ihre wertenden Kriterien schöpfen. Nicht jeder natürlich, der eine Rezension schreibt, muss ein Literaturwissenschaftler sein, oder gar ein Universitätsprofessor. In dieser Branche gab man, historisch gesehen, sogar jenen Kritikern den Vorzug, die einen unmittelbaren Zugang zur Praxis der Gegenwart hatten. Anz und Baasner, die Herausgeber der im Rahmen des Marburger Projekts 2004 entstandenen Literaturkritik. Geschichte - Theorie- Praxis, notieren dazu:

Denn Literaturkritik im modernen Sinn, auch das zeigt der Blick auf ihre Geschichte, entstand erst aus der - oft polemischen - Distanz zur akademischen Gelehrsamkeit im Umgang mit Literatur. Gelehrte jedoch waren die meisten Kritiker, die es zu hohem Ansehen brachten, selbst. Lessing, Herder, die Brüder Schlegel, Georg Lukacs oder Walter Benjamin konnten sich in ihrem historischen und theoretischen Wissen mit ihren hochgelehrten Zeitgenossen an den Universitäten ohne weiteres messen. Aber sie waren weltoffener als diese, bei allen historischen Kenntnissen ihrer Gegenwart stärker zugewandt; ihr Engagement galt den Möglichkeiten zukünftiger Literatur. Nicht zuletzt konnten sie so schreiben, daß sie nicht nur in ihren eigenen Kreisen verstanden wurden (Anz und Baasner, 2007).

Also müsste jeder, der eine wertende Literaturkritik schreibt, wie es auch Anz und Baasner festhalten, wenn er auch nicht ein beruflicher Literaturwissenschaftler ist, eine hohe Fachkompetenz aufweisen. Und das ist vorerst schon ein gutes Kriterium, um auch im Internet eine Selektion in Bezug auf eine gute/schlechte Literaturkritik zu treffen. In der gegenwärtigen Internetzeit, dem Zeitalter der Digitalisierung, sind die Grundkriterien für eine gute Rezension, z. B. autorisierte Texte ${ }^{1}$, die Fachkompetenz der unterzeichneten Namen und eine umfassende Darstellung der Texte, die von Information, über Interpretation bis Wertung mit einer wissenschaftlichen Argumentationsweise hinreichen, eher verschwommen. Man spricht in Bezug auf die Online-Literaturkritik von einer Demokratisierung (Radojčić, 2009), auch von einer Volkes Stimme (Domsch, 2003), die sich einer gründlicheren Gelehrsamkeit nicht mehr verpflichtet fühlt. Sicherlich hat der Boom der Online-Publikationen auch Vorteile mit sich gebracht, so einen schnellen Zugriff für den Nutzer/Leser, die Aktualität der Texte, die oft den Printmedien einen Schritt voraus sind, da sie augenblicklich die Information verbreiten, ferner die Bequemlichkeit des von Zu-Hause-ausRecherchierens, die jederzeitige Verfügbarkeit auch der alten Ressourcen (es genügt ein Mausklick, um in die alten Archive zu gelangen), aber auch die Freiheit für

\footnotetext{
${ }^{1}$ Im Gegensatz zu Texten, die unter den echten Namen von Autoren veröffentlicht und so autorisiert werden, erscheint im Internet eine Vielzahl von anonymen Texten oder Texten, die unter einem Decknamen publiziert wurden.
} 
potenzielle Hobbykritiker, ihre Buchbesprechungen ohne Umstände und die strengen Regeln der Kulturredaktionen zu veröffentlichen, und zwar kostenlos. So genügt es, einen privaten Blog zu starten, um vor aller Öffentlichkeit mit einer Buchbesprechung präsent zu sein. Die Konsequenz dieser Demokratisierung ist ein unüberschaubares Angebot an verschiedenen Online-Publikationen von literaturkritischen Texten, die von Laientexten bis zu hochkompetenten Buchbesprechungen hinreichen. So ist der ambivalente Charakter des Phänomens der Demokratisierung von OnlinePublikationen vor unseren Augen nur allzu offensichtlich. Der serbische Literaturwissenschaftler und Dichter Saša Radojčić hält folgende Antinomien in Bezug auf diese Ambivalenz fest: dem Individuellen sei das Private entgegengesetzt, dem Liberalen das Anarchische, dem Kommunikativen das Oberflächliche (2009). Ferner führt Radojčić aus, dass nur wenige der zur Verfügung stehenden Internetportale nur ein wenig strengeren literaturästhetischen Kriterien standhalten würden. Um einen Überblick und eine Selektion in Bezug auf die Qualität der Online-Texte zu sichern, empfiehlt dieser Autor, die wertenden Kriterien herauszukristallisieren, die bei dieser unüberschaubaren Zahl von verschiedenen Blogs und Portalen mit literaturkritischen Ansprüchen eine Auswahl ermöglichen würden (2009).

Auf diese Thesen bezugnehmend wird in dem vorliegenden Beitrag am Korpus von verschiedenen Blogs und Portalen mit literaturkritischen Texten versucht, eine Selektion zu treffen. Dabei wurden die folgenden Schwerpunkte in den Fokus gestellt: das redaktionelle Konzept mit Zielen der Veröffentlichung von literaturkritischen Texten, Autorisierung der Online-Publikationen, Fachkompetenz der unterzeichneten Namen, die Beschaffenheit der Texte (die eine Selektion, Information, Interpretation und Wertung aufweisen sollten) und schließlich der Kontrollmechanismus seitens der Redaktion. Anhand dieser Kriterien haben wir folgende Arten von Websites geprüft: private Blogs, Rezensionsforen, Online- Zeitschriften und Magazine, Buchhandlungsportale, die auch Buchbesprechungen anbieten, und literaturkritische Portale.

Bei der Analyse von privaten Blogs hat sich gezeigt, dass in Bezug auf genannte Kriterien die unverlässlichen diejenigen sind, die Hobbyrezensenten im eigenen Arrangement publizieren, aber dies ohne nähere biografische Daten und fachliche Konzepte der dargebotenen Literaturkritik. Bei einer unüberschaubaren Zahl von solchen privaten Blogs sind an dieser Stelle nur einzelne Beispiele zur Illustration herausgelöst, z. B. der private Blog mojaKritika.com, der die genannten Kriterien nicht erfüllen würde. Auf diesem Blog ist der Name des Autors angegeben, dies jedoch ohne 
nähere Informationen zu persönlichen Daten, inklusive einer professionellen Biografie. Die angebotenen Texte beschränken sich auf eine Wertung ohne differenzierbare Argumentationsweise. Ein Grundkonzept fehlt, und stattdessen wird nur ein Motto in Form eines kurzen Gedankensplitters eingeblendet. Die Kontaktdaten, eine übliche Grundinformation, fehlen ebenso.

Ein entgegengesetztes Beispiel in Bezug auf die Qualität der veröffentlichten Texte auf privaten Blogs ist der Blog des serbischen Literaturwissenschaftlers Saša Šmulja. ${ }^{2}$ Auch hier handelt es sich um einen privaten Blog, aber mit ausführlichen Daten zur akademischen Laufbahn des Autors. Die angebotenen Literaturkritiken weisen eine hohe Fachkompetenz auf, die an den literaturwissenschaftlichen Kriterien zu erkennen ist, die in den Texten angewandt werden. Der Vorteil eines solchen Blogs ergibt sich somit aus dem Umstand, dass die Texte autorisiert sind, dass der Nutzer Zugang zu biografischen Daten hat, und dass auch eine hohe Fachkompetenz sowohl aus der professionellen akademischen Laufbahn als auch aus wertenden Kriterien bei der Beurteilung der literarischen Werke hervorgeht. Ein Nachteil dieses Blogs ist, dass er nicht permanent aktualisiert wird.

Als ein weiteres ähnliches Beispiel ist der private Blog des Germanisten Christian Köllerer ${ }^{3}$ zu nennen, auf dem autorisierte Texte erscheinen und auch biografische Daten zugänglich sind, die bezeugen, dass der Autor als Germanist Fachwissen aufweist und die Texte dementsprechend fachlich bearbeitet sind. Ein zusätzlicher Vorteil dieses Blogs ist die Zusammenstellung einer privaten Bibliothek, die für den Nutzer abrufbar ist sowie die regelmäßige Aktualisierung der Seite. Die Texte werden auch archiviert, so dass sie jederzeit zur Verfügung stehen.

Innerhalb dieser Online-Form ist auch der Blog von Nico Haase zu erwähnen ${ }^{4}$; ein Hobbyrezensent, der aus seiner privaten Website eine Institution entwickelt hat, und zwar dank der Unterstützung der Mitarbeiter aus mehreren Berufsfeldern, die engagiert werden. So ist der private Blog zu einem Gruppenblog geworden. Der Vorteil dieser Seite ist, dass autorisierte Texte erscheinen, doch die Texte ihrer Beurteilung von literarischen Werken nach massenorientiert sind. Davon zeugen die Kompetenzen der Mitarbeiter, die meistens keine profilierten Wissenschaftler sind, auch keine Berufskritiker (Hobbyschreiber, Studierende, Menschen, die im beruflichen Leben, z. B. Archäologie, Psychologie, Kultur, praktische Berufserfahrungen gesammelt haben

\footnotetext{
${ }^{2}$ Vgl. http://smulja.blogspot.ba (Stand: 10.04.2017).

${ }^{3}$ Vgl. www.koellerer.net (Stand: 12.04.2017).

${ }^{4} \mathrm{Vgl}$. buchtipps.net (Stand: 13.04.2017).
} 
...) wie auch der Umstand, dass oft Trivialliteratur besprochen wird, z.B. ScienceFiction oder Krimis.

www.booklover.com ist ein weiterer Blog, der keine näheren Daten zur Seite enthält (z.B. Gründungsziele, Kontakt). Die Buchbesprechungen sind Texte, die nicht autorisiert sind. Die Kriterien bei der Beurteilung der Bücher sind subjektiv gefärbt, ohne eine nachvollziehbare Argumentationsweise. Sie bieten eher eine Information und Inhaltswiedergabe an als wertende Kriterien. Als Laientexte sind diese Kritiken massenorientiert.

Ähnliches bietet der Blog nightfliersbookspace.blogspot.com an. Die Texte sind nicht autorisiert, die Namen der Rezensionsautoren sind chiffriert, z. B. High Duke. Trivialliteratur steht im Vordergrund, vor allem Science-Fiction. Die Buchbesprechungen beschränken sich auf Information und eine laienhafte Inhaltswiedergabe.

Die Rezensionsforen als weitere Gruppe von Online-Publikationen, z. B. http://www. serbiancafe.com, www.literaturforum.de, etc. weisen eine große Palette von Nutzern auf und sind massenorientiert. Manche Texte sind autorisiert, manche nicht. Sie dienen vor allem der Gestaltung eines Kommunikationskanals, bei dem Meinungsaustausch und Diskussion zur angebotenen Literatur ermöglicht werden. Die Diskussionen entsprechen aber eher einem mündlichen Austausch ohne feste grammatikalische Regeln in den Äußerungen und sie sind selten fachlich untermauert. Der Vorteil solcher Formen ist aber, dass sie durch Diskussionsforen die Idee eines kommunikativen Online-Austauschkanals in Gang gesetzt haben, wodurch man in Bezug auf die Tendenzen in der Literaturentwicklung und auf die Bedürfnisse der breiten Masse aufmerksam gemacht werden kann. Einen solchen OnlineKommunikationskanal haben die seriöseren literaturwissenschaftlich orientierten Websites in kompetente Diskussionsforen verwandelt, die durch Meinungsaustausch nicht nur den Literaturkritikern von Nutze sind, sondern auch für die Leser eine Orientierung in Bezug auf Kriterien und Tendenzen anbieten.

Buchbesprechungen bieten auch Online-Kulturmagazine an. Sie zeigen sich verlässlich hinsichtlich ihres informativen Charakters. Kaleidoskop-media.com ist ein solches Online- Magazin, das durch das dargebotene Konzept eine gute Informationsquelle für den Leser/Nutzer darstellt. Es sind komplette Daten über die Redaktion abrufbar. Alle Texte sind mit vollem Namen unterzeichnet. Es handelt sich vor allem um journalistische Buchbesprechungen, die keinen Anspruch auf eine tiefere literaturwissenschaftliche Analyse aufweisen. Ihr Ziel ist, den Leser über das kulturelle 
Leben auf dem Laufenden zu halten. Dementsprechend sind die Texte meistens von Journalisten verfasst und erfüllen damit ihre angestrebte Funktion, die Buchbesprechung als Information zu lancieren, nicht als Wertung. Solche Seiten eignen sich für den Nutzer gut dazu, eine Selektion unter Dargebotenem in der Bücherwelt zu treffen, und darüber Grundinformationen zu sammeln. Unter deutschen Anbietern ist Perlentaucher.de zu nennen, welcher ein ähnliches Konzept fördert. Von Thierry Chervel gegründet ist dieses Kulturmagazin auf die Darbietung einer guten Kulturübersicht orientiert. Ein zusätzlicher Vorteil dieses Magazins, das auf einem klaren informativen Konzept fußt, ist, dass es regelmäßig über die Rezensionen in Tageszeitungen informiert (z. B. FAZ, SZ, Die Welt, Die Zeit, etc), und dass auch journalistische Stellungnahmen zu diesen Rezensionen bezogen werden, bzw. eine Zweitverwertung von Zeitungsrezensionen. Das Archiv des Perlentauchers bietet weiterhin einen Überblick über die veröffentlichten und meistdiskutierten Bücher. Obwohl nicht literaturwissenschaftlich, sondern journalismusorientiert, bieten solche Magazine innerhalb von Grundkriterien eines literaturkritischen Textes, also Selektion, Information, Interpretation und Wertung, eine verlässliche Quelle in Bezug auf die ersten zwei Kriterien an.

Die produktivsten Websites mit Buchbesprechungen sind Online-Buchhändler, z. B. der schon weltbekannte Amazon.de. Außer dem Verkauf ermöglichen sie ihren Kunden, die eigenen Buchbesprechungen zu veröffentlichen, bzw. Stellungnahmen, die meistens in Form eines Kurztextes auf der Website unter den Bewertungen erscheinen. Diese Texte haben einen kommerziellen Zweck und tragen zur Werbung bei. Sie operieren auf der Oberfläche mit Grundkriterien wie Selektion, Information und Wertung, weisen dabei aber zum großen Teil eine laienhafte Schreibweise auf. Die meisten Wertungen sind subjektiv gefärbt und massenorientiert. Dass solche marktorientierten Kritiken bis zum extrem kommerziell Ausgerichteten reichen können, ist aus dem Beispiel des Literaturwissenschaftlers und Online-Redakteurs Oliver Pfohlmann zu entnehmen, der Literaturkritiken im Internet schon jahrelang erforscht. Er nennt das folgende Beispiel:

Versehentlich outet Amazon in den USA die Internetadressen Tausender ihrer Rezensenten, und sieh da: Hinter ,A Reader from Chicago' oder ,New York' verbirgt sich nicht selten der Autor selbst, der eine anonyme Selbstrezension publizierte. Das dürfte hierzulande wenig anders sein. (Pfohlmann, 2007).

Eine gute Übersicht über das literarische Leben bieten aber die Internetportale an, gegründet von Literaten, Berufskritikern oder Menschen, die eine reichliche 
Erfahrung im praktischen literarischen Leben haben, z. B. in Verlagen, Buchhandlungen, etc. Das Portal knjizevnost.org ist ein gutkonzipiertes Portal mit autorisierten Texten. Für eine gute Übersicht sorgen auch die differenziert gestalteten Menüs und Untermenüspaletten, in denen Literaturkritiken, Kolumnen und Interviews abrufbar sind. Dieses Portal veröffentlicht ebenso literarische Texte, selektiert nach Gattung. Eine E-Bibliothek und das Archiv bieten dem Nutzer zusätzlich die Gelegenheit, sich die besprochenen Bücher, auch die in archivierten Rezensionen thematisierten, anzuschauen. Da sie neben Wertung auch Information im Fokus haben, werden die Informationen zu Ausschreibungen, z. B. für Preisverleihungen täglich aktualisiert. Die literaturkritischen Texte werden von Journalisten und Literaturwissenschaftlern unterzeichnet, auch mit Daten zur professionellen Biografie. Die Felder, die für die Kommentare der Nutzer freigehalten werden, ermöglichen einen kommunikativen Austauschkanal.

Unter deutschen Websites verfügt das Portal literaturcafe.de über ein ähnliches Konzept. Das gut arrangierte Portal wurde von Wolfgang Tischer, einem Buchhändler mit jahrelanger Erfahrung im literarischen Leben, gegründet. Solche Portale haben den Vorteil, dass sie sich aufgrund von Auslassung der strikt wissenschaftlich orientierten Texte, die eher für einen engen Kreis der Leser nachzuvollziehen wären, an ein breiteres Publikum wenden, diesem aber gleichzeitig Informationen zu guter Literatur zugänglich machen, die mit namhaften Preisverleihungen in Zusammenhang steht und von renommierten Berufskritikern, Literaturwissenschaftlern oder Kulturjournalisten besprochen wird. Somit stellen sie in Bezug auf die Frage, wie man auch in der breiteren Masse für eine wertvolle Literatur werben kann, eine gute Möglichkeit dar, durch eine ausbalancierte, dem breiteren Kreis von Nutzern angepasste Schreibweise, die Qualität mit dem Interessewecken zu vereinbaren.

Die strikt literaturwissenschaftlich orientierten Portale haben sich seit den 1990 er-Jahren auch im Internet profiliert. Sie bieten literaturkritische Texte an, die von Personen mit einem umfangreichen Fachwissen unterzeichnet werden, sind aber dadurch für einen schmaleren Kreis der potenziellen Besucher geeignet, die selbst professionelle Kompetenzen aufweisen. Unter solchen Portalen findet man z.B. literaturkritik.de und IASL online.

Literaturkritik.de wurde von Thomas Anz gegründet, dem Literaturwissenschaftler und Leiter des TransMIT-Zentrums für Literaturvermittlung in den Medien an der Universität Marburg. Die Literaturkritiken auf diesem Portal verfassen Mitarbeiter, die selbst Literaturwissenschaftler sind, oder Studierende, die 
in Seminaren für die Literaturvermittlung an der Universität Marburg Erfahrungen sammeln. Eine ausführliche Liste mit Namen sowie den biografischen Daten der Mitarbeiter ist abrufbar. Die literaturkritischen Texte erscheinen monatlich und sind auch nach Themen sortiert. Im Archiv sind sie nach Titeln oder Autorennamen abrufbar. Durch Untermenü-Navigation kann der Nutzer auch in die Bestenliste bzw. Bestsellerliste gelangen. Das Portal bietet zusätzlich eine Übersicht über das kulturelle Leben an, eine journalistische Ecke, die unter dem Reiter "Kulturjournal" abrufbar ist. Die freien Felder für Leserbriefe bieten zusätzlich Raum für literarische Diskussionen an. Das seit jeher bekannte Problem bezüglich der Disziplin der Literaturkritik, dass ihr nämlich für ihre Leistungen nie eine adäquate finanzielle Entlohnung zur Verfügung stand, versucht dieses Portal durch marktorientierte Geschäftsregeln zu lösen. Neben informativen und literaturkritischen Texten wurden auch ein eigener Verlag und eine Buchhandlung gegründet. Außerdem bietet man den Abonnenten die Möglichkeit, ihre Rezensionen ohne Absprache mit der Redaktion zu veröffentlichen, ein Schritt, der das literaturwissenschaftliche Konzept in Richtung des Kommerziellen auflockert. In diesem Zusammenhang steht auch die marktorientierte Geschäftsregel, die den Abonnenten einen freien Zugang zum gesamten System ermöglicht, inklusive der Veröffentlichung der eigenen Texte, dem Zugang zu Volltexten und gedruckten Ausgaben, dem Zugang zu kostenlosen Lexika etc.

Ein rein literaturwissenschaftliches Konzept ohne marktorientierte Prätentionen bietet das Portal IASL online an, auf dem aber ausschließlich Fachliteratur besprochen wird. Auf diesem Portal erscheinen hochwertige Literaturkritiken, die von universitären Professoren verfasst werden. Angegeben ist eine ausführliche Liste von Mitarbeitern und ihr professioneller Hintergrund. Ein kommunikativer Austausch wird auch ermöglicht, und zwar durch thematische Foren und Beiträge. Damit wird auch das letzte Grundkriterium einer guten Literaturkritik, dass sie nämlich neben Selektion, Information, Interpretation und Wertung auch zur Diskussion über literarisches Leben anregen sollte, erfüllt. Die Qualität der Texte, die die Fachliteratur ausschließlich aufgrund ausdifferenzierter literaturwissenschaftlicher Kriterien beurteilen, steht für dieses Portal an oberster Stelle. Diese Website ist nicht marktorientiert und das Kommerzielle ist zugunsten der Qualität völlig zurückgestellt. Das Kriterium der Kontrolle, nach dem sich der Leser über die Qualität der Texte versichern kann, ist auf diesem Portal auch erfüllt. Die veröffentlichten Texte, obwohl von hochkompetenten Mitarbeitern verfasst, werden selbst einem Begutachtungsverfahren unterzogen. Die Besucher dieses Portals sind Menschen, die selbst eine hohe Fachkompetenz im 
Bereich Literaturwissenschaft aufweisen. Eine breitere Öffentlichkeit ist durch dieses professionelle Konzept des Portals, auf dem ausschließlich die Fachliteratur von Fachkennern besprochen wird, ausgeschlossen. Somit erfüllt IASL online inhaltlich alle aufgestellten Kriterien, die für eine verlässliche Literaturkritik bürgen, allerdings mit dem Umstand, dass nur ein engerer Kreis von Besuchern diese Dienstleistungen in Anspruch nehmen kann.

Aus der vorliegenden Analyse hat sich ergeben, dass die Online-Literaturkritik eine breite Palette von verschiedenen Arten der Buchbesprechung anbietet. Dabei reichen die Texte von Laienkritiken bis hin zu professionellen Buchbesprechungen. Das heißt ferner, dass das Internet als ein alternatives Medium die Selektion zwischen Laienhaftem und Professionellem nicht mehr fokussiert. Im Vergleich zu den Printmedien, welche die Fachkompetenz und Auswahl von wertvollen Texten in den Vordergrund stellen, hat das Internet eine Demokratisierung der Publizierung mit sich gebracht. Allerdings sind die professionellen Rezensionen unabhängig von der Demokratisierung der Veröffentlichung nicht verloren gegangen. Der Nutzer kann im Internet, je nach eigenen Bedürfnissen, eine Auswahl treffen. Es zeigt sich dabei, dass die Websites, auf denen professionelle Mitarbeiter ohne marktorientierten Anspruch die Rezensionen verfassen, auch für die Qualität der dargebotenen Texte bürgen. Bei Online-Buchhandlungen hat sich ergeben, dass das kommerziell Ausgerichtete in einem unmittelbaren Zusammenhang mit dem Laienhaften steht. Dieser Umstand, in Hinblick auf die Tatsache, dass zum Teil auch kompetente Buchbesprechungen erscheinen, bedarf einer weiteren Untersuchung. Meistens gilt aber: Je marktorientierter eine Plattform, desto weniger ist Fachwissen gefragt. Noch extremer trifft dieses Fazit auf die Portale zu, auf denen die Autoren gegen ein entsprechendes Entgelt eine positive Rezension verfasst bekommen. Solche Portale sind in diesem Beitrag nicht einzeln untersucht worden.

Die vorliegende Analyse hat weiterhin gezeigt, dass die Auseinandersetzung zwischen Hochliteratur und Unterhaltungsliteratur auch im Medium Internet weiter ausgetragen wird. So erweist sich auch das umgekehrte Fazit als wahr: Je literaturwissenschaftlicher konzipiert, desto weniger Besucher nehmen diese Dienstleistungen in Anspruch.

Wenn man das gesellschaftliche Ziel der Literaturkritik - die ästhetische Erziehung einer breiteren Masse - in den Fokus stellt, so zeigen sich die Portale wie Online-Magazine als gut konzipiert, um die Sympathien einer breiteren Leserschaft für gute Literatur zu gewinnen. Sie wenden sich mit einer ausbalancierten Schreibweise 
an den Nutzer, die unterhält und zugleich belehrt, und werben damit um einen größeren Besucherkreis. Die anspruchsvolleren Online-Buchbesprechungen bekommen wiederum aufgrund des Umstands, dass auf diese Seiten oft durch Zufall eine unüberschaubare Zahl von Menschen gelangt, die Chance von einem breiteren Leserkreis wahrgenommen zu werden, während in der Zeit der Printmedien immer nur die Sucher nach gezielt fachlich orientierten literaturkritischen Rubriken die potenziellen Rezipienten waren.

\section{Literaturverzeichnis}

\section{Primärinternetquellen}

www.mojaKritika.com (Stand: 10.04.2017).

www.smulja.blogspot.ba (Stand: 10.04.2017).

www.koellerer.net (Stand: 12.04.2017).

www.buchtipps.net (Stand: 13.04.2017).

www.booklover.com (Stand: 13.04.2017).

www.nightfliersbookspace.blogspot.com (Stand: 13.04.2017).

www.serbiancafe.com (Stand: 20.04.2017).

www.literaturforum.de (Stand: 21.04.2017).

www.kaleidoskop-media.com (Stand: 29.04.2017).

www.perlentaucher.de (Stand: 02.05.2017)

www.amazon.de (Stand: 10.05.2017)

www.knjizevnost.org (Stand: 13.05.2017)

www.literaturcafe.de (Stand: 13.05.2017)

www.literaturkritik.de (Stand: 18.05.2017)

www.iaslonline.de (Stand: 20.05.2017)

\section{Sekundärliteratur}

Anz, T., Baasner, R. (2007). Literaturkritik. Geschichte - Theorie - Praxis. München: Becksche Reihe.

Domsch, S.(2003). Die Kundenflüsterer. TAZ, 27.12.2003, 21.

Pfohlmann, O. (2005). Kleines Lexikon der Literaturkritik. Marburg: Literaturwissenschaft.de. 
Pfohlmann, O. (2007). Literaturkritik in der Bundesrepublik. In Anz, T., Baasner, R. (Hg.) Literaturkritik. Geschichte - Theorie - Praxis. (S. 160-194). München: Becksche Reihe.

Radojčić, S. (2009). Srpska književna blogosfera [Die serbische literarische BlogSphäre]. Kultura, (124), 61-68.

\section{Summary \\ LITERARY CRITICISM ONLINE AN ANALYSIS OF THE EVALUATION CRITERIA ON GERMAN AND SERBIAN INTERNET PORTALS}

This paper investigates literary reviews on both German and Serbian Internet portals, which predominantly shifted to the on-line sphere during the last two decades, thus becoming one step ahead of the literary reviews in the printed media where they are less and less published, apart from being provided with less and less space. The investigation of literary reviews on the selected portals relies on analytic and comparative methods, with regards to those evaluative criteria that ensure good critical review and that are relied upon during discussion of the literary texts. The paper is examining whether the authorized texts are published at these portals and to what extent, whether the signed authors show professional competence, whether scientific criteria are among prerequisites in the published texts, whether the professional competence is susceptible to external factors, such as pressure from publishing houses, and whether they are market-oriented and governed by business rules. Finally, the paper investigates if the reviews are subjected to further revision process, as means of a control mechanism. The analysis of the abovementioned factors should give us a better insight in how the requirements for good literary reviews are met on the Internet portals.

Keywords: Online literary criticism, blogs, forums, online journals and magazines, bookstore portals, literary criticism portals. 